\title{
Kinetic Monte Carlo theory of sliding friction
}

\author{
Octavio Javier Furlong, ${ }^{1}$ Sergio Javier Manzi, ${ }^{2}$ Victor Daniel Pereyra, ${ }^{2}$ Victor Bustos, ${ }^{2}$ and Wilfred T. Tysoe ${ }^{1, *}$ \\ ${ }^{1}$ Department of Chemistry and Biochemistry and Laboratory for Surface Studies, \\ University of Wisconsin-Milwaukee, Milwaukee, Wisconsin 53211, USA \\ ${ }^{2}$ Instituto de Física Aplicada (INFAP), CONICET-Universidad Nacional de San Luis, Chacabuco 917, 5700 San Luis, Argentina
}

(Received 21 May 2009; revised manuscript received 12 August 2009; published 7 October 2009)

\begin{abstract}
The sliding friction as a function of scanning velocity at the nanometer scale was simulated based on a modified one-dimensional Tomlinson model. Monte Carlo theory was exploited to describe the thermally activated hopping of the contact atoms, where both backward and forward jumps were allowed to occur. By comparing with the Monte Carlo results, improvements to current semiempirical solutions [E. Riedo et al., Phys. Rev. Lett. 91, 084502 (2003)] were made. Finally, experimental results of sliding friction on a $\mathrm{NaCl}(100)$ as a function of normal load and scanning velocity [E. Gnecco et al., Phys. Rev. Lett. 84, 1172 (2000)] where successfully simulated.
\end{abstract}

DOI: $10.1103 /$ PhysRevB.80.153408

PACS number(s): 68.35.Af, 62.20.Qp, 02.70.Uu

Introduction. One of the conceptually simplest models that addresses the atomic origins of sliding friction was initially proposed by Tomlinson and Prandtl, ${ }^{1,2}$ which assumes a periodically and sinusoidally varying change in potential energy at the sliding interface. This is most often applied to the case of a single asperity contact, such as might be encountered in atomic force microscope (AFM) experiments. ${ }^{3,4}$ This model has been extensively applied to understanding the temperature and velocity dependence of sliding friction, as well as the stick-slip behavior observed in this type of experiment. ${ }^{5-10}$ In the model, a harmonic strain caused by the motion of the contact modifies the sinusoidal sliding potential. In the simplest case of such a model (at zero temperature), sliding occurs when the barrier height of the total (harmonic + sinusoidal) potential decreases to zero, resulting in atomic stick-slip motion. However, temperature and velocity effects are easily included by incorporating a Boltzmann probability that an atom surmounts the potential barrier before it decreases to zero. In this case, the velocity dependence arises since the potential becomes time dependent so that the rate at which the tip surmounts the barrier also depends on time. In order to analytically solve the equation for the Tomlinson model, the height of the barrier close to the inflection point is approximated by a simple analytical function.

However, such kinetic events can also be naturally described by Monte Carlo theory. A similar approach has been previously exploited ${ }^{11-14}$ in order to study temperature effects on the slip position (stick-slip motion) as well as on the frictional force. Here we focus on the velocity dependence of the frictional force. In order to test whether this approach is feasible, Monte Carlo calculations have been performed for a one-dimensional Tomlinson model at finite temperature. The results of the simulation are in excellent agreement with previous analytical solutions of the Tomlinson model with a sinusoidal sliding potential. ${ }^{7}$ However, the advantage of using such simulations is that it can generally be applied to any form of the periodic sliding potential. In addition, the ability to rapidly obtain numerical solutions to the Tomlinson model allows more precise approximations to be made to the semi- empirical solution and this is illustrated below. Finally the simulation results are compared with the experimental AFM data obtained by Gnecco et al.,$^{5}$ on a $\mathrm{NaCl}(100)$ surface using a silicon tip, where the frictional force was measured as a function of normal load and scanning velocity.

Theoretical methods. The Tomlinson model involves a time-dependent harmonic potential superimposed on a sinusoidal potential describing the contact. The time-dependent potential can be expressed as

$$
V(x, t)=-\frac{E_{0}}{2} \cos \left(\frac{2 \pi x}{a}\right)+\frac{k_{L}}{2}(v t-x)^{2} .
$$

The first term describes the periodic potential of the surface and the second term is due to the imposed harmonic potential, where $k_{L}$ is the effective stiffness of the contact, $a$ is the surface lattice constant, $E_{0}$ is the potential corrugation, $x$ is the position of the tip, and $v$ is the scanning velocity so that $v t$ becomes the time-dependent position of the tip support $X$. In the simplest expression of the model, the system is assumed to be at temperature $T=0$. Under this condition, sliding takes place at some critical force $F^{*}$ at which the potential barrier height $\Delta E$ becomes zero. Thus, $F^{*}$ is the lateral force required to slide the contact at zero temperature. The system then jumps to the next energy minimum resulting in a decrease in the lateral force, giving rise to the experimentally observed stick-slip motion. Since at $T=0$, the first and second derivatives of the potential must be zero when sliding takes place, one can easily obtain $F^{*}$, which is

$$
F^{*}=\frac{\pi E_{0}}{a} .
$$

At some finite temperature $T$, assuming that the jump is thermally assisted as the instantaneous lateral force $F_{L}$ approaches $F^{*}$, this results in a theoretical velocity and temperature-dependent friction force that agrees well with experiment. Since the transition over the potential barrier is considered to be a thermally activated process, the transition rate $w$ can be described by 


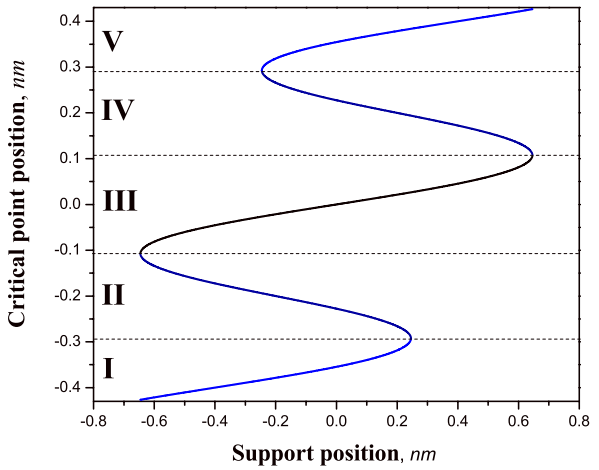

FIG. 1. (Color online) Plot of the input matrix that is used for the Monte Carlo simulations (see text) where the critical-point position for sliding is plotted versus the support position.

$$
w(t)=f_{0} \exp \left[-\frac{\Delta E(t)}{k_{B} T}\right],
$$

where $f_{0}$ is the frequency attempt of a transition and $k_{B}$ is the Boltzmann constant. Monte Carlo methods are ideally suited to analyzing these types of processes.

For each Monte Carlo trial the value of $w$ is calculated at some time $t$ and compared to a random number $\xi_{1}$ uniformly distributed in the interval $(0,1)$. If $\xi_{1}<w$ the transition is allowed to occur, where both backward and forward transitions are allowed. Finally the lateral force is recorded as a function of time. The process is repeated a sufficient number of times to yield an average friction force with negligible statistical error. The conversion between Monte Carlo time and real time is made by defining an elementary transition probability per unit time. ${ }^{15}$

Monte Carlo simulations usually consist of repeating a process under a certain probability distribution and calculating the average of all the outcomes. To minimize the calculation time, it is useful to initially define all the parameters that will remain constant during the simulation. In our case, these parameters are temperature, velocity, pre-exponential factor (attempt frequency), potential height, lateral force constant, and lattice spacing. Furthermore, the positions of the critical points of the potential, its minima and maxima, shift as the lateral force varies. In order to avoid calculating them during each step, a position matrix is generated prior to the simulation. Since only the first derivative of the Tomlinson potential is needed to obtain the matrix, the only required parameters are the potential corrugation $E_{0}$, the lattice constant $a$, and the lateral stiffness constant $k_{L}$. To be consistent with the simulations shown below the parameters used are $E_{0}=0.466 \mathrm{eV}, a=0.4 \mathrm{~nm}$, and $k_{L}=0.86 \mathrm{~N} / \mathrm{m}$ and the results for the matrix are displayed in Fig. 1. As indicated by Eq. (1), the form of the sliding potential varies as the cantilever position changes (with time). This results in a change in the height of the potential, which has a time-dependent decrease from $E_{0}$. Figure 2 illustrates how each critical point is affected as the support position increases.

Comparison between Monte Carlo theory simulation and analytical results. In order to test the validity of the Monte Carlo simulation, the results are compared with a semiempir-

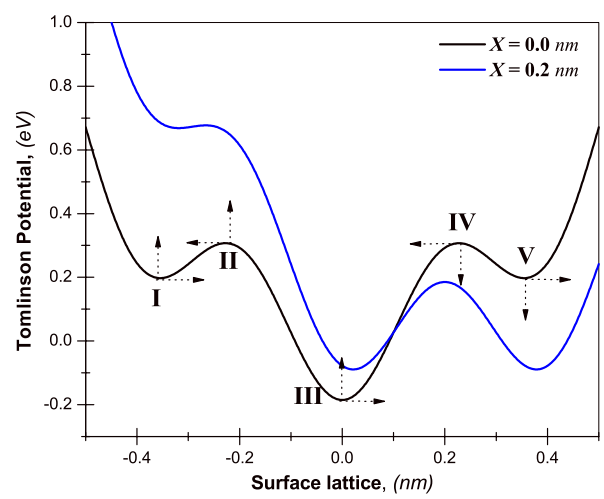

FIG. 2. (Color online) Plot of the shape of the combined sinusoidal and harmonic Tomlinson potential for an undisplaced support $(X=0.0 \mathrm{~nm})$ and for a displaced support $(X=0.2 \mathrm{~nm})$. This shows both the change in height of the potential as a function of displacement as well as the change in positions of the maxima and minima. Also indicated are the positions in the potential corresponding to the values in the matrix plotted in Fig. 1.

ical equation that allows an analytical solution to the Tomlinson model to be obtained when using a sinusoidal potential. In order for the particle to jump from one position $x_{\text {min }}$ to the next, it overcomes an energy barrier located at $x_{\max }$ so that $\Delta E$ is given by

$$
\Delta E=V\left(x_{\max }, t\right)-V\left(x_{\min }, t\right) .
$$

At zero temperature, the lateral force required to provide the energy for the jump is the critical force $F^{*}$ and is given by Eq. (2). At some finite temperature, however, the transition of the tip from one minimum to the adjacent one becomes a thermally activated process, and its occurrence is given by a Gaussian distribution of probabilities. In this case, the probability of the particle not jumping $p(t)$ is described by the master Eq. (5),

$$
\frac{d p}{d t}=-f_{0} \exp \left[-\frac{\Delta E(t)}{k_{B} T}\right] p(t),
$$

where the parameters are defined above. Gnecco et al. ${ }^{5}$ observed that the logarithmic velocity dependence of the lateral sliding force could be explained by assuming a linear relationship between $\Delta E$ and the lateral force $F_{L}$. This logarithmic velocity dependence occurs over a limited and relatively low range of velocities but it disappears when the jumps occur very close to the critical point given by $\Delta E=0$ and this effect is not captured by a linear relationship between $\Delta E$ and $F_{L}$. To address this issue, Sang et al. ${ }^{6}$ proposed an improved relationship, shown in Eq. (6),

$$
\Delta E=\frac{1}{\beta}\left(F^{*}-F_{L}\right)^{3 / 2},
$$

where the parameter $\beta$ is a constant of the system. Using this approximation for $\Delta E$, Riedo et $a{ }^{7}{ }^{7}$ were able to derive a semiempirical expression for the velocity dependence of the lateral frictional force $F_{L}$ but without limitations to the velocity range as 


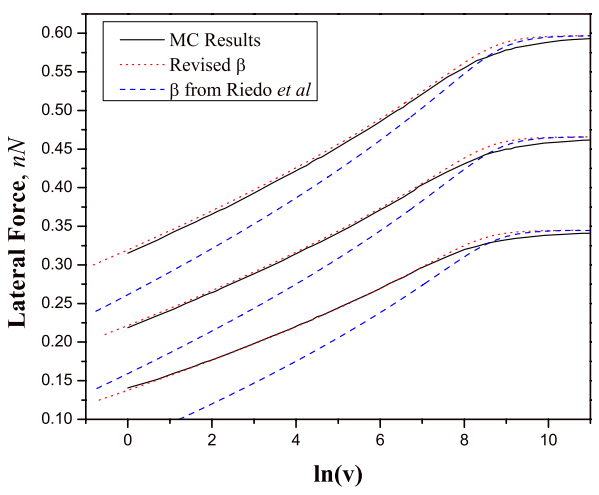

FIG. 3. (Color online) Comparison of the lateral force $F_{L}$ versus $\ln (v)[$ Eq. (7)] using the value of $\beta$ from Riedo et al. (dashed lines) compared with the Monte Carlo simulations (solid lines) using the same parameters for the sliding potential. Shown also as dotted line are the results calculated using Eq. (7) with the improved formula for the value of $\beta$, Eq. (14).

$$
\frac{1}{\beta k_{B} T}\left(F^{*}-F_{L}\right)^{3 / 2}=\ln \frac{v_{0}}{v}-\frac{1}{2} \ln \left(1-\frac{F_{L}}{F^{*}}\right),
$$

where $v_{0}$ is equal to

$$
v_{0}=\frac{2 f_{0} \beta k_{B} T}{3 k_{L} \sqrt{F^{*}}} .
$$

Note that these formulas are correct only for the simplest sinusoidal potential in the Tomlinson model. In order to test the validity of using Monte Carlo methods to simulate Tomlinson sliding, the Monte Carlo results are compared with those of Eq. (7) using the formula for $\beta$ given in reference. ${ }^{7}$ The results are compared in Fig. 3 where the lateral force $F_{L}$ is plotted as a function of $\ln (v)$, for various values of $E_{0}(0.275,0.370$, and $0.475 \mathrm{eV})$, which therefore specify the values of $F^{*}$ as $0.345,0.466$, and $0.597 \mathrm{nN}$, respectively. The remaining values used for the calculations and simulations are $f_{0}=50 \mathrm{MHz}, k_{L}=0.86 \mathrm{~N} / \mathrm{m}$, and $a=0.4 \mathrm{~nm}$. These parameters were chosen since they are relevant to sliding on $\mathrm{NaCl}(100)$ single-crystal surface as will be discussed below. The resulting lateral force versus velocity curves obtained using the Monte Carlo method are displayed in Fig. 3 as solid lines with the top curve corresponding to the highest value of $E_{0}$ (of $0.475 \mathrm{eV}$ ) and the bottom curve to the lowest value $(0.275 \mathrm{eV})$. The semiempirical analytical results, Eq. (7), were then calculated using the expression for $\beta$ from Riedo et al., ${ }^{7}$ which is defined entirely by the properties of the surface potential. This gives rise to the results plotted as dashed lines in Fig. 3 and clearly do not agree with the results of the Monte Carlo simulations. The disparity between the Monte Carlo simulations and the semiempirical calculations was traced to the approximate value of $\beta$ and a more precise value was calculated (see next section). The results of the solution to the semiempirical equation [Eq. (7)] using the improved formula for $\beta$ is also plotted as dotted lines and is in much better agreement with the results of the Monte Carlo simulations. There are, however, some slight differences between the two curves, in particular, in the region where sliding undergoes a transition from velocity- dependent to velocity-independent sliding. We believe this disparity arises since the semiempirical equation [Eq. (7)] does not take into account effects due to the decreasing distance between adjacent minima and maxima as sliding occurs while the Monte Carlo calculation does. We feel therefore that the Monte Carlo results provide a more accurate representation of the solution to the Tomlinson model.

Improved calculation of the $\beta$ parameter. As noted in the previous section, it was found that the value of the $\beta$ parameter from Riedo et al. ${ }^{7}$ yielded an analytical solution that did not agree with the results of the Monte Carlo simulations. In their work, $\beta$ was calculated by taking the first derivative of the Tomlinson potential with respect to $x$. By expanding in a Taylor's series around the critical point $x^{*}=a / 4$, it is found that $\Delta E$ varies as a function of time as

$$
\Delta E=\frac{2 \sqrt{2}}{3} E_{0}\left(1-\frac{k_{L} v t}{F^{*}}\right)^{3 / 2} \text {. }
$$

Assuming the lateral force to be $F_{L} \approx k_{L} v t$ and comparing with Eq. (6) yields

$$
\beta_{\sin }=\frac{3 \pi \sqrt{F^{*}}}{2 \sqrt{2} a} .
$$

However, the Monte Carlo simulations reveal that, by considering that $F_{L} \approx k_{L} v t$, the assumption that the minima and maxima of the interaction potential remain fixed in position under any lateral force introduces an error that, depending on the values of the input parameters, can result in significant differences, as emphasized by the data in Fig. 3. In order to obtain a more accurate expression for $\beta$, a different approach is taken in the following. First consider the case when $F_{L}$ $=0$ at $t=0$. In this case, Eq. (6) becomes

$$
\Delta E=\frac{1}{\beta}\left(F^{*}\right)^{3 / 2}
$$

and Eq. (4) can be rewritten as

$$
\begin{aligned}
\Delta E= & -\frac{E_{0}}{2} \cos \left(\frac{2 \pi x_{\max }}{a}\right)+\frac{k_{L}}{2}\left(v t-x_{\max }\right)^{2} \\
& +\frac{E_{0}}{2} \cos \left(\frac{2 \pi x_{\min }}{a}\right)-\frac{k_{L}}{2}\left(v t-x_{\min }\right)^{2} .
\end{aligned}
$$

At $t=0, x_{\max }=a / 2$ and $x_{\min }=0$, and substituting in Eq. (12), $\Delta E$ becomes

$$
\Delta E=\frac{k_{L} a^{2}}{8}+E_{0} .
$$

Finally substituting Eq. (13) into Eq. (11) and replacing $E_{0}$ with the value for $F^{*}$ from Eq. (2) yields a more precise value for $\beta$ for a sinusoidal potential as

$$
\beta_{\sin }=\frac{\left(F^{*}\right)^{3 / 2}}{\left(\frac{k_{L} a^{2}}{8}+\frac{F^{*} a}{\pi}\right)} .
$$

This new expression for $\beta$ also includes the effect of $k_{L}$, which from our Monte Carlo simulations can clearly have an important effect on $\beta$ (Fig. 4). 


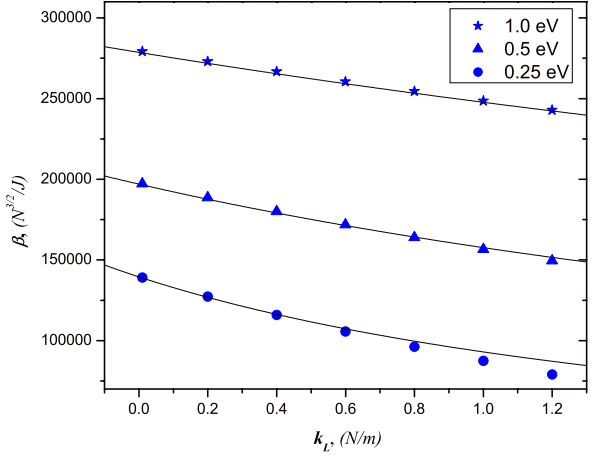

FIG. 4. (Color online) Plot of the value of $\beta$ versus the cantilever force constant $k_{L}$ for various values of the height of the Tomlinson potential $E_{0}$ of $1.0,0.5$, and $0.25 \mathrm{eV}$ calculated using Eq. (14) (shown as solid line) or by using Monte Carlo theory (symbols)

Comparison with experimental results. In order to further test the validity of the Monte Carlo simulations, these are used to simulate the experimental results obtained by Gnecco et $a .^{5}$ In these experiments, the frictional force was measured by AFM with a silicon tip sliding against a $\mathrm{NaCl}(100)$ surface, under ultrahigh-vacuum conditions. The frictional force was measured as a function of scanning velocity under two different normal loads of 0.65 and $0.44 \mathrm{nN}$ at room temperature. The experimental results are compared with the simulations in Fig. 5, where the agreement between the simulations and the experimental results is very good. The fit was obtained using parameters that are consistent with the experimental conditions. Thus, the lattice spacing was maintained at $a=0.4 \mathrm{~nm}$, in agreement with the lattice constant of $\mathrm{KCl}$, the effective lateral force constant $k_{L}$ was $0.86 \mathrm{~N} / \mathrm{m}$ and the temperature $T$ maintained at $293 \mathrm{~K}$. In order to obtain the best fit to the experimental data, the potential-energy corrugation $E_{0}$ was set to $0.275 \mathrm{eV}$ at a normal load of $0.65 \mathrm{nN}$ and $0.37 \mathrm{eV}$ at a normal load of $0.44 \mathrm{nN}$, and the attempt frequency $f_{0}$ was set to $50 \mathrm{MHz}$. Sang et al. ${ }^{6}$ obtained similar values for the fitting parameters by solving a Langevin equation for Tomlinson sliding. These results indicate that Monte Carlo simulations can be efficiently and effectively used to mimic sliding in the Tomlinson model. An advantage of using the Monte Carlo approach is that it can also be simply

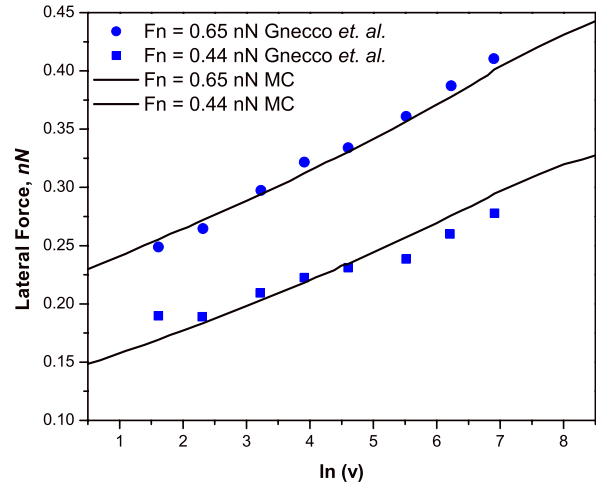

FIG. 5. (Color online) Plots of the experimentally measured lateral force versus $\ln (v)$, where $v$ is the sliding velocity at normal loads of $0.65(-)$ and $0.44 \mathrm{nN}(\mathbf{\square})$ for sliding on $\mathrm{NaCl}(100)$. Shown as solid lines are the solutions to the Tomlinson sliding model obtained from Monte Carlo simulations using the following parameters: $a=0.4 \mathrm{~nm}, k_{L}=0.86 \mathrm{~N} / \mathrm{m}$, and $T=293 \mathrm{~K}$. $E_{0}=0.475 \mathrm{eV}$ at a normal load of $0.65 \mathrm{nN}$ and $0.353 \mathrm{eV}$ at a normal load of $0.44 \mathrm{nN}$, and $f_{0}=50 \mathrm{MHz}$

applied to periodic sliding potentials of any shape and is not restricted just to simple sinusoidal potentials.

Conclusions. A one-dimensional Tomlinson model was analyzed using Monte Carlo theory to explore the velocity and load dependence of sliding friction at the nanometer scale. Since temperature effects are those that give rise to a velocity-dependent frictional force, Monte Carlo theory was used to include the thermal contribution to the hopping process of the atoms in the contact. To test the validity of the Monte Carlo simulations, our results were compared with a semiempirical equation proposed by Riedo et al., ${ }^{7}$ using the same parameters for the interaction potential. It was found that the semiempirical equation could be improved and a new expression for the parameter $\beta$ was obtained. To further test the validity of the simulations, experimental results obtained by $\mathrm{AFM}$ on a $\mathrm{NaCl}(100)$ surface using a tungsten tip were successfully reproduced.

We gratefully acknowledge support for this work by the National Science Foundation under Grant No. CMMI 0826151 .
*Author to whom correspondence should be addressed. FAX: (414) 229-5036; wtt@uwm.edu

${ }^{1}$ G. A. Tomlinson, Philos. Mag. 7, 905 (1929).

${ }^{2}$ L. Prandtl, Z. Angew. Math. Mech. 8, 85 (1928).

${ }^{3}$ R. W. Carpick and M. Salmeron, Chem. Rev. 97, 1163 (1997).

${ }^{4}$ E. Gnecco, R. Bennewitz, T. Gyalog, and E. Meyer, J. Phys.: Condens. Matter 13, R619 (2001).

${ }^{5}$ E. Gnecco, R. Bennewitz, T. Gyalog, Ch. Loppacher, M. Bammerlin, E. Meyer, and H. J. Güntherodt, Phys. Rev. Lett. 84, 1172 (2000).

${ }^{6}$ Y. Sang, M. Dubé, and M. Grant, Phys. Rev. Lett. 87, 174301 (2001).

${ }^{7}$ E. Riedo, E. Gnecco, R. Bennewitz, E. Meyer, and H. Brune, Phys. Rev. Lett. 91, 084502 (2003).
${ }^{8}$ C. Fusco and A. Fasolino, Phys. Rev. B 71, 045413 (2005).

${ }^{9}$ A. Socoliuc, R. Bennewitz, E. Gnecco, and E. Meyer, Phys. Rev. Lett. 92, 134301 (2004).

${ }^{10}$ M. Porto, V. Zaloj, M. Urbakh et al., Tribol. Lett. 9, 45 (2000).

${ }^{11}$ M. Dienwiebel, N. Pradeep, G. S. Verhoeven, H. W. Zandbergen, and J. W. M. Frenken, Surf. Sci. 576, 197 (2005).

${ }^{12}$ D. G. Abel, S. Y. Krylov, and J. W. M. Frenken, Phys. Rev. Lett. 99, 166102 (2007).

${ }^{13}$ S. Y. Krylov and J. W. M. Frenken, J. Phys.: Condens. Matter 20, 354003 (2008).

${ }^{14}$ K. B. Jinesh, S. Y. Krylov, H. Valk, M. Dienwiebel, and J. W. M. Frenken, Phys. Rev. B 78, 155440 (2008).

${ }^{15}$ J. L. Sales, R. O. Uñac, M. V. Gargiulo, V. Bustos, and G. Zgrablich, Langmuir 12, 95 (1996). 